\title{
РЕГУЛЯТОРЫ УСПОКОЕНИЯ РЕШЕНИЯ ЛИНЕЙНЫХ СИСТЕМ НЕЙТРАЛЬНОГО ТИПА
}

\author{
(C) 2016 г. .В. Метельский, В. Е. Хартовский, О. И. Урбан
}

\begin{abstract}
Для линейных автономных систем нейтрального типа с соизмеримыми запаздываниями в состоянии и управлении решена задача успокоения решения регулятором с обратной связью по состоянию. При этом замкнутая система становится системой нейтрального типа с конечным спектром. Отличительной чертой исследований является допущение отсутствия у исходной системы свойства полной управляемости.
\end{abstract}

DOI: $10.1134 / \mathrm{S} 0374064116030122$

\section{ВВЕДЕНИЕ}

Для линейных автономных дифференциально-разностных систем запаздывающего типа критерий спектральной управляемости [1] является необходимым и достаточным для разрешимости задачи назначения системе конечного спектра [2-4]. В работах [5-7] для таких систем с одним входом при условии спектральной управляемости строились регуляторы, обеспечивающие замкнутой системе полное успокоение, конечный спектр и асимптотическую устойчивость. В работе [8] предлагался регулятор с обратной связью переменной структуры, обеспечивающий успокоение решения многовходной системы запаздывающего типа с соизмеримыми запаздываниями в состоянии и управлении, при этом свойство спектральной управляемости не являлось необходимым. Обобщение задачи Красовского об успокоении системы с запаздыванием [9] для различных классов систем проведено в работах [10-13].

В настоящей работе решается задача построения регулятора с обратной связью по состоянию, обеспечивающего успокоение решения многовходной линейной автономной дифференциально-разностной системы нейтрального типа с соизмеримыми запаздываниями. Отличительной чертой предлагаемого исследования является возможность его реализации для не полностью управляемых объектов $[13,14]$, что для систем запаздывающего типа равнозначно отсутствию свойства спектральной управляемости. Предложенный регулятор как функция состояния системы имеет переменную структуру, что позволяет эффективно использовать его не только для успокоения решения $[8,15]$, но и для решения задачи модальной управляемости [16].

\section{1. ПОСТАНОВКА ЗАДАЧИ}

Объектом исследования является линейная автономная дифференциально-разностная система нейтрального типа с соизмеримыми запаздываниями

$$
\dot{x}(t)-\sum_{i=1}^{m} D_{i} \dot{x}(t-i h)=\sum_{i=0}^{m}\left(A_{i} x(t-i h)+B_{i} u(t-i h)\right), \quad t>0,
$$

где $x$ - вектор непрерывного кусочно-дифференцируемого решения, $u$ - вектор кусочнонепрерывного управления, $D_{i} \in \mathbb{R}^{n \times n}, \quad i=\overline{1, m}, \quad A_{i} \in \mathbb{R}^{n \times n}, \quad B_{i} \in \mathbb{R}^{n \times r}, i=\overline{0, m} ; \quad h>0-$ постоянное запаздывание. Начальное условие для системы $\left(1^{\prime}\right) \quad x(t)=\eta(t), u(t)=u^{0}(t)$, $t \in[-m h, 0]$, функции $\eta(t), u^{0}(t), \quad t \in[-m h, 0]$, предполагаются непрерывной кусочнодифференцируемой и кусочно-непрерывной соответственно. 
Обозначим $D(\lambda)=\sum_{i=1}^{m} D_{i} \lambda^{i}, \quad A(\lambda)=\sum_{i=0}^{m} A_{i} \lambda^{i}, \quad B(\lambda)=\sum_{i=0}^{m} B_{i} \lambda^{i}, \quad E_{k} \in \mathbb{R}^{k \times k}$ - единичная матрица, $\lambda$ - оператор сдвига $\left(\lambda^{k} f(t)=f(t-k h)\right)$ и запишем исходную систему $\left(1^{\prime}\right)$ в виде, более удобном для дальнейшего изложения:

$$
\left(E_{n}-D(\lambda)\right) \dot{x}(t)=A(\lambda) x(t)+B(\lambda) u(t), \quad t>0 .
$$

Под задачей успокоения решения системы (1) будем понимать задачу выбора управления $u(t), \quad t>0$, такого, что

$$
x(t) \equiv 0, \quad t \geq t_{1}
$$

где $t_{1}>0$ - некоторый фиксированный момент времени (один и тот же для всех начальных условий). Цель исследования - замкнуть систему (1) регулятором с обратной связью по состоянию $u=u(x(t), \ldots, x(t-\varepsilon h), \dot{x}(t-h), \ldots, \dot{x}(t-\varepsilon h))(\varepsilon \in \mathbb{N}$ - некоторое натуральное число) так, чтобы выполнялось тождество (2).

Регулятор, обеспечивающий тождество (2), будем строить в виде трех последовательно соединенных контуров, первый из них описывается уравнениями

$$
u(t)=T \psi(t)+v^{1}(t), \quad \psi(t)=S \psi(t-h)+v^{2}(t), \quad t>0,
$$

второй контур - уравнениями

$$
v(t)=\dot{y}(t)+w^{1}(t), \quad \dot{y}(t)=L^{x}(\lambda) \dot{x}(t)+L^{y}(\lambda) \dot{y}(t)+w^{2}(t), \quad t>0,
$$

а третий контур - уравнениями

$$
\begin{gathered}
w(t)=M_{1}^{x}(\lambda) x(t)+M_{1}^{y}(\lambda) y(t)+M_{1}^{z}(\lambda) z(t), \\
\dot{z}(t)=M_{2}^{x}(\lambda) x(t)+M_{2}^{y}(\lambda) y(t)+M_{2}^{z}(\lambda) z(t), \quad t>0,
\end{gathered}
$$

где

$$
\begin{gathered}
T \in \mathbb{R}^{r \times r_{T}}, \quad S \in \mathbb{R}^{r_{T} \times r_{T}}, \quad v=\operatorname{col}\left[v^{1}, v^{2}\right], \quad v^{1} \in \mathbb{R}^{r}, \quad v^{2} \in \mathbb{R}^{r_{T}}, \\
y=\operatorname{col}\left[y^{1}, y^{2}\right], \quad y^{1} \in \mathbb{R}^{r}, \quad y^{2} \in \mathbb{R}^{r_{T}}, \quad w=\operatorname{col}\left[w^{1}, w^{2}\right], \quad w^{i} \in \mathbb{R}^{r_{1}}, \quad i=1,2, \\
L^{x}(\lambda) \in \mathbb{R}^{r_{1} \times n}[\lambda], \quad L^{y}(\lambda) \in \mathbb{R}^{r_{1} \times r_{1}}[\lambda], \quad L^{x}(0)=0_{r_{1} \times n}, \quad L^{y}(0)=0_{r_{1} \times r_{1}}
\end{gathered}
$$

(здесь и далее $0_{k_{1} \times k_{2}} \in \mathbb{R}^{k_{1} \times k_{2}}-$ нулевая матрица),

$$
\begin{gathered}
z \in \mathbb{R}^{s}, \quad M_{1}^{x}(\lambda) \in \mathbb{R}^{2 r_{1} \times n}[\lambda], \quad M_{1}^{y}(\lambda) \in \mathbb{R}^{2 r_{1} \times r_{1}}[\lambda], \quad M_{1}^{z}(\lambda) \in \mathbb{R}^{2 r_{1} \times s}[\lambda], \\
M_{2}^{x}(\lambda) \in \mathbb{R}^{s \times n}[\lambda], \quad M_{2}^{y}(\lambda) \in \mathbb{R}^{s \times r_{1}}[\lambda], \quad M_{2}^{z}(\lambda) \in \mathbb{R}^{s \times s}[\lambda]
\end{gathered}
$$

$\left(\mathbb{R}^{k_{1} \times k_{2}}[\lambda]-\right.$ множество матриц размера $k_{1} \times k_{2}$, элементы которых являются полиномами переменной $\lambda$ с действительными коэффициентами), $r_{T}, r_{1}=r+r_{T}$ - некоторые натуральные числа. Считаем, что функции $x(t), t \leq m h, y(t), z(t), t \leq 0$, и $\psi(t), t \leq 0$, любые непрерывные кусочно-дифференцируемые и кусочно-непрерывная соответственно.

Поясним, что будем понимать под системой (1), замкнутой регулятором (3)-(5). Обозначим

$$
X=\operatorname{col}[x, y, z], \quad S_{3}(\lambda)=\left[M_{2}^{x}(\lambda), M_{2}^{y}(\lambda), M_{2}^{z}(\lambda)\right]
$$

и запишем матрицу

$$
S_{1}(\lambda)=\left[M_{1}^{x}(\lambda), M_{1}^{y}(\lambda), M_{1}^{z}(\lambda)\right]
$$

в виде

$$
S_{1}(\lambda)=\operatorname{col}\left[S^{u}(\lambda), S^{\psi}(\lambda), S_{2}(\lambda)\right]
$$


где матрица $S^{u}(\lambda)$ - первые $r$ строк матрицы $S_{1}(\lambda), S^{\psi}(\lambda)$ - следующие $r_{T}$ строк и $S_{2}(\lambda)-$ оставшиеся строки матрицы $S_{1}(\lambda)$. Подставим переменную $w$, определяемую первым уравнением в (5), в (4), после этого полученную величину $v$ подставим в (3) (т.е. исключим из равенств (3)-(5) функции $v$ и $w)$. В итоге регулятор (3)-(5) запишем в виде

$$
\begin{gathered}
u(t)=\dot{y}^{1}(t)+S^{u}(\lambda) X(t)+T \psi(t), \quad \psi(t)=S \psi(t-h)+\dot{y}^{2}(t)+S^{\psi}(\lambda) X(t), \\
\dot{y}(t)=L^{x}(\lambda) \dot{x}(t)+L^{y}(\lambda) \dot{y}(t)+S_{2}(\lambda) X(t), \quad \dot{z}(t)=S_{3}(\lambda) X(t), \quad t>0 .
\end{gathered}
$$

При любой начальной функции $\psi(t), t \leq 0$, на каждом полуинтервале $(k h,(k+1) h]$, $k=0,1, \ldots$, функция $\psi(t)$ выражается через величины $X(\tau), \dot{y}^{2}(\tau), \tau \leq t$, согласно второму (разностному) уравнению в (6). После ее подстановки в первое уравнение в (6) получим функцию $u(t), t \in(k h,(k+1) h]$, зависящую от $X(\tau), \dot{y}(\tau), \tau \leq t$, которая в совокупности с двумя последними уравнениями в (6) определяет регулятор на полуинтервале $(k h,(k+1) h]$. Замыкая им систему (1), на полуинтервале $(k h,(k+1) h], k=0,1, \ldots$, получаем линейную дифференциально-разностную систему нейтраліьного типа с соизмеримыми запаздываниями. На следующем полуинтервале описанный процесс повторяется. Определенную таким образом при $t>0$ систему будем называть системой (1), замкнутой регулятором (6) (или, что то же самое, регулятором (3)-(5)), а под ее решением будем понимать функцию $X(t), t>0$. Отсюда также следует, что на каждом полуинтервале $(k h,(k+1) h], k=0,1, \ldots$, регулятор $(6)$ как функция состояния $X$ замкнутой системы изменяет свою структуру. Далее покажем, что, несмотря на переменную структуру регулятора (6), замкнутая система (1), (6) при $t>m h$ будет линейной автономной дифференциально-разностной системой нейтрального типа $c$ coизмеримыми запаздываниями и конечным спектром.

Задача 1. Требуется указать условия существования регулятора (6) и схему замыкания им системы (1) так, чтобы для решения замкнутой системы (1), (6) выполнялось тождество (2) независимо от начального условия.

Замечание 1. Если для любого начального условия системы (1) существует управление $u(t), t \in\left(0, t_{1}-m h\right]$, обеспечивающее тождество (2) при условии $u(t) \equiv 0, t>t_{1}-m h$, то система $(1)$ называется $[13,14]$ полностью управляемой. Обратим внимание, что в задаче 1 требование $u(t) \equiv 0, t>t_{1}-m h$, отсутствует.

Замечание 2. Критерий полной управляемости системы (1) определяется [13, 14] одновременным выполнением условий

$$
\operatorname{rank}\left[p\left(E_{n}-D\left(e^{-p h}\right)\right)-A\left(e^{-p h}\right), B\left(e^{-p h}\right)\right]=n, \quad p \in \mathbb{C}, \quad \operatorname{rank}\left[E_{n}-D(\lambda), B(\lambda)\right]=n, \quad \lambda \in \mathbb{C},
$$

где $\mathbb{C}$ - множество комплексных чисел. Далее будет показано, что условия существования регулятора (6) являются более слабыми по сравнению с критерием полной управляемости.

\section{2. ОСНОВНОЙ РЕЗУЛЬТАТ}

Для формулировки основного результата нам понадобятся следующие обозначения. По аналогии с работами $[8,10-13,15,16]$ рассмотрим последовательность векторов $\delta_{k}$, $k=m, m+1, \ldots$, которая является решением разностного уравнения

$$
B_{0} \delta_{k}+\sum_{i=1}^{m} B_{i} \delta_{k-i}=0, \quad k=m, m+1, \ldots,
$$

порождаемого начальным условием $\delta_{i}=\tilde{\delta}_{i}, i=\overline{0, m-1}$. Последовательность $\delta_{k}, k=$ $=m, m+1, \ldots$, определяемая уравнением $(7)$, существует в том и только в том случае [10], когда $\tilde{\delta}_{m-i}=T_{i} c, i=\overline{1, m}$, где $T_{i} \in \mathbb{R}^{r \times r_{T}}$ - некоторые матрицы, $c \in \mathbb{R}^{r_{T}}$ - произвольный постоянный вектор (один и тот же для всех матриц $T_{i}$ ). Метод построения матриц $T_{i}$ приведен в работах $[10,12,13]$, поэтому здесь его не описываем. Отметим, что его реализация всегда возможна и заключается в решении конечного числа однородных алгебраических 
систем. В соотношениях (6) и далее полагаем $T=T_{m}$. Матрицу $S \in \mathbb{R}^{r_{T} \times r_{T}}$ регулятора (6) определим как решение системы уравнений

$$
B_{0} T_{1} S+\sum_{i=1}^{m} B_{i} T_{i}=0_{n \times r_{T}}, \quad T_{k} S=T_{k-1}, \quad k=\overline{2, m}
$$

разрешимость которой следует из определения матриц $T_{i}$. Заметим, что выполняется равенство $\sum_{i=0}^{m} B_{i} T S^{m-i}=0_{n \times r_{T}}$.

Определим матрицы $\tilde{B}_{0}=B_{0} T, \quad \tilde{B}_{i}=\tilde{B}_{i-1} S+B_{i} T, \quad i=\overline{1, m}$. Заметим, что $\tilde{B}_{m}=$ $=\sum_{i=0}^{m} B_{i} T S^{m-i}=0_{n \times r_{T}}$. Обозначим

$$
\tilde{B}(\lambda)=\sum_{i=0}^{m} \tilde{B}_{i} \lambda^{i}, \quad G(\lambda)=[B(\lambda), \tilde{B}(\lambda)]=\sum_{i=0}^{m} G_{i} \lambda^{i}
$$

где $G_{i}=\left[B_{i}, \tilde{B}_{i}\right], \quad i=\overline{0, m}$.

Теорема 1. Задача 1 разреиима тогда и только тогда, когда одновременно выполнялотся следующие условия:

$$
\begin{gathered}
\operatorname{rank}\left[p\left(E_{n}-D\left(e^{-p h}\right)\right)-A\left(e^{-p h}\right), G\left(e^{-p h}\right)\right]=n, \quad p \in \mathbb{C} \\
\operatorname{rank}\left[E_{n}-D(\lambda), G(\lambda)\right]=n, \quad \lambda \in \mathbb{C} .
\end{gathered}
$$

Доказательство. Необходимость. Условия (8), (9) являются [13] необходимыми для существования программного управления $u(t), t>0$, удовлетворяющего тождеству (2) для любого начального условия системы (1). Значит, они необходимы и для существования соответствующего управления типа обратной связи.

Достаточность условий (8), (9) следует из алгоритма синтеза регулятора (6), который приведен и обоснован в п. 3.

\section{3. АЛГОРИТМ СИНТЕЗА РЕГУЛЯТОРА (6)}

3.1. Синтез первого контура. Первый контур регулятора строится так, чтобы: 1) свести исходную систему (1) к системе (1), (3) с новым управлением $v$, которая в отличие от исходной системы (1) обладает свойством полной управляемости; 2) обеспечить принадлежность замкнутой системы (1), (6) к классу линейных автономных дифференциально-разностных систем нейтрального типа с соизмеримыми запаздываниями. Покажем, что матрицы $T$ и $S$, входяцие в структуру первого контура регулятора (6) и выбранные в п. 2, обеспечивают указанные свойства.

Лемма 1. Пусть управление $u(t), t>0$, определяется соотношениями (3), a $x(t)$, $t \in[0, m h]$, - решение системы (1)-(3). Для того чтобы фулкиия $x(t), t>m h$, являлась решением системы (1), (3) при $t>m h$, необходимо и достаточно, чтобы она удовлетворяла cuстеме

$$
\left(E_{n}-D(\lambda)\right) \dot{x}(t)=A(\lambda) x(t)+B(\lambda) v^{1}(t)+\tilde{B}(\lambda) v^{2}(t), \quad t>m h .
$$

Доказательство. Рассмотрим следующую цепочку равенств:

$$
\begin{aligned}
& B(\lambda) T \psi(t)=B_{0} T \psi(t)+\sum_{i=1}^{m} B_{i} \lambda^{i} T \psi(t)=\tilde{B}_{0} \psi(t)+\sum_{i=1}^{m}\left(\tilde{B}_{i}-\tilde{B}_{i-1} S\right) \lambda^{i} \psi(t)= \\
& =\sum_{i=0}^{m} \tilde{B}_{i} \lambda^{i} \psi(t)-\sum_{i=1}^{m} \tilde{B}_{i-1} S \lambda^{i} \psi(t)=\sum_{i=0}^{m-1} \tilde{B}_{i} \lambda^{i}\left(E_{r_{T}}-S \lambda\right) \psi(t)=\tilde{B}(\lambda) v^{2}(t) .
\end{aligned}
$$

Пусть $x(t), t>m h,-$ решение системы (1)-(3). Для доказательства необходимости условия леммы следует в систему (1) подставить управление, определяемое равенствами (3), и учесть 
приведенную выше цепочку равенств. Для доказательства достаточности надо повторить эти же рассуждения, но в обратном порядке. Лемма доказана.

Приведем

Пример 1. Уравнение $\dot{x}(t)-\dot{x}(t-h)=u(t)-u(t-h), t>0$, не обладает свойством полной управляемости. Пусть первый контур регулятора имеет вид

$$
u(t)=\psi(t)+v^{1}(t), \quad \psi(t)=\psi(t-h)+v^{2}(t), \quad t>0 .
$$

Тогда, исключив функцию $\psi$, получим уравнение $\dot{x}(t)-\dot{x}(t-h)=v^{1}(t)-v^{1}(t-h)+v^{2}(t)$, $t>h$, которое определяет функцию $x(t), t>h$, и обладает свойством полной управляемости.

Рассмотрим регулятор (6). Обозначим $S_{\psi}^{u}(\lambda)=\operatorname{col}\left[S^{u}(\lambda), S^{\psi}(\lambda)\right]$.

Следствие 1. Для того чтобы функчия $X(t), t>m h$, была решением системъ (1), (6), необходимо и достаточно, чтобы она удовлетворяла линейной автономной дифференииально-разностной системе нейтрального типа с соизмеримыми запаздъваниями

$$
\begin{gathered}
\left(E_{n}-D(\lambda)\right) \dot{x}(t)=A(\lambda) x(t)+G(\lambda)\left(\dot{y}(t)+S_{\psi}^{u}(\lambda) X(t)\right), \\
\dot{y}(t)=L^{x}(\lambda) \dot{x}(t)+L^{y}(\lambda) \dot{y}(t)+S_{2}(\lambda) X(t), \\
\dot{z}(t)=S_{3}(\lambda) X(t), \quad t>m h .
\end{gathered}
$$

Замечание 3. В качестве матрицы $G(\lambda)$ можно взять линейно-независимые столбцы матрицы $[B(\lambda), \tilde{B}(\lambda)]$, которые предварительно можно привести к более простому виду с помоцью элементарных преобразований.

3.2. Синтез второго контура. Второй контур строится так, чтобы обеспечить замкнутой системе (1), (3), (4) характеристический квазиполином запаздывающего типа. Этого можно добиться, если выбрать матрицы $L^{x}(\lambda), L^{y}(\lambda)$, удовлетворяющими тождеству

$$
\operatorname{det}\left[E_{n_{r}}-\check{D}(\lambda)\right] \equiv 1
$$

где $\check{D}(\lambda)=\left[\begin{array}{cc}D(\lambda) & G(\lambda) \\ L^{x}(\lambda) & L^{y}(\lambda)\end{array}\right], n_{r}=n+r_{1}$. Обоснуем возможность такого выбора.

Лемма 2. Для того чтобы существовали полиномиальные матрицы $L^{x}(\lambda)=\sum_{j=1}^{m} L_{j}^{x} \lambda^{j}$, $L^{y}(\lambda)=\sum_{j=1}^{m} L_{j}^{y} \lambda^{j}, \quad L_{j}^{x} \in \mathbb{R}^{r_{1} \times n}, L_{j}^{y} \in \mathbb{R}^{r_{1} \times r_{1}}, L^{x}(0)=0_{r_{1} \times n}, \quad L^{y}(0)=0_{r_{1} \times r_{1}}$, обеспечивающие выполнение тождества (11), необходимо и достаточно выполнения условия (9).

Доказательство. Доказательство необходимости затруднений не доставляет, поэтому не приводится. Докажем достаточность. Определим матрицы

$$
\begin{gathered}
\widehat{D}=\left[\begin{array}{cccccccc}
D_{1} & \ldots & D_{m-1} & D_{m} & G_{1} & \ldots & G_{m-1} & G_{m} \\
E_{n} & \ldots & 0_{n \times n} & 0_{n \times n} & 0_{n \times r_{1}} & \ldots & 0_{n \times r_{1}} & 0_{n \times r_{1}} \\
\ldots \ldots \ldots \ldots \ldots \ldots \ldots \ldots \ldots \ldots \ldots \ldots \ldots \ldots \ldots & \ldots \ldots & \ldots \ldots & \ldots \ldots \\
0_{n \times n} & \ldots & E_{n} & 0_{n \times n} & 0_{n \times r_{1}} & \ldots & 0_{n \times r_{1}} & 0_{n \times r_{1}} \\
0_{r_{1} \times n} & \ldots & 0_{r_{1} \times n} & 0_{r_{1} \times n} & 0_{r_{1} \times r_{1}} & \ldots & 0_{r_{1} \times r_{1}} & 0_{r_{1} \times r_{1}} \\
0_{r_{1} \times n} & \ldots & 0_{r_{1} \times n} & 0_{r_{1} \times n} & E_{r_{1}} & \ldots & 0_{r_{1} \times r_{1}} & 0_{r_{1} \times r_{1}} \\
\ldots \ldots \ldots \ldots & \ldots \ldots \ldots \ldots \ldots \ldots \ldots \ldots \\
0_{r_{1} \times n} & \ldots & 0_{r_{1} \times n} & 0_{r_{1} \times n} & 0_{r_{1} \times r_{1}} & \ldots & E_{r_{1}} & 0_{r_{1} \times r_{1}}
\end{array}\right], \\
\widehat{G}=\operatorname{col}\left[G_{0}, 0_{(m-1) n \times r_{1}}, E_{r_{1}}, 0_{(m-1) r_{1} \times r_{1}}\right] .
\end{gathered}
$$

Сначала докажем, что из условия (9) следует, что

$$
\operatorname{rank}\left[E_{m n_{r}}-\widehat{D} \lambda, \widehat{G}\right]=m n_{r}, \quad \lambda \in \mathbb{C} .
$$

Предположим противное: условие (12) нарушается при некотором $\lambda=c_{0} \in \mathbb{C}\left(c_{0} \neq 0\right)$. Тогда найдется ненулевой вектор $q=\operatorname{col}\left[q_{1}, \ldots, q_{2 m}\right], q_{i} \in \mathbb{C}^{n}, i=\overline{1, m}, q_{i} \in \mathbb{C}^{r_{1}}, \quad i=\overline{m+1,2 m}$, такой, что

$$
q^{\prime}\left(E_{m n_{r}}-\widehat{D} c_{0}\right)=0_{1 \times m n_{r}}, \quad q^{\prime} \widehat{G}=0_{1 \times r_{1}}
$$


где штрих обозначает операцию транспонирования. Положим

$$
\begin{gathered}
E_{*}(\lambda)=\operatorname{col}\left[E_{n}, \lambda E_{n}, \ldots, \lambda^{(m-1)} E_{n}, 0_{r_{1} \times n}, \ldots, 0_{r_{1} \times n}\right], \quad E_{*} \in \mathbb{C}^{m n_{r} \times n}, \\
E_{* *}(\lambda)=\operatorname{col}\left[0_{n \times r_{1}}, \ldots, 0_{n \times r_{1}}, E_{r_{1}}, \lambda E_{r_{1}}, \ldots, \lambda^{(m-1)} E_{r_{1}}\right], \quad E_{* *} \in \mathbb{C}^{m n_{r} \times r_{1}} .
\end{gathered}
$$

В силу равенств (13)

$$
\begin{gathered}
q^{\prime}\left(E_{m n_{r}}-\widehat{D} c_{0}\right) E_{*}\left(c_{0}\right)=q^{\prime}\left(E_{n}-D\left(c_{0}\right)\right)=0_{1 \times n}, \\
q^{\prime}\left(E_{m n_{r}}-\widehat{D} c_{0}\right) E_{* *}\left(c_{0}\right)=-q_{1}^{\prime}\left(\sum_{i=1}^{m} G_{i}\left(c_{0}\right)^{i}\right)+q_{m+1}^{\prime}=0_{1 \times r_{1}}, \\
q_{1}^{\prime} G_{0}+q_{m+1}^{\prime}=0_{1 \times r_{1}} .
\end{gathered}
$$

Последние три равенства противоречат условию (9). Равенство (12) доказано.

Из условия (12) следует, что

$$
\operatorname{rank}\left[\widehat{G}, \widehat{D} \widehat{G}, \ldots, \widehat{D}^{n_{r} m-1} \widehat{G}\right]=\operatorname{rank}\left[\widehat{G}, \widehat{D} \widehat{G}, \ldots, \widehat{D}^{n_{r} m-1} \widehat{G}, \widehat{D}^{n_{r} m}\right] .
$$

Тогда в силу равенства (14) найдется матрица $L^{x y}=\left[L_{1}^{x}, \ldots, L_{m}^{x}, L_{1}^{y}, \ldots, L_{m}^{y}\right], \quad L_{i}^{x} \in \mathbb{R}^{r_{1} \times n}$, $L_{i}^{y} \in \mathbb{R}^{r_{1} \times r_{1}}, i=\overline{1, m}$, такая, что имеет место тождество

$$
\operatorname{det}\left[E_{m n_{r}}-\left(\widehat{D}+\widehat{G} L^{x y}\right) \lambda\right] \equiv 1
$$

Действительно, пусть $\operatorname{rank}\left[\widehat{G}, \widehat{D} \widehat{G}, \ldots, \widehat{D}^{n_{r} m-1} \widehat{G}\right]=\rho$. Построим матрицу $Q, \operatorname{det} Q \neq 0$, такую, что

$$
Q^{-1} \widehat{D} Q=\left[\begin{array}{cc}
\widehat{D}_{1}^{Q} & \widehat{D}_{2}^{Q} \\
0_{\left(m n_{r}-\rho\right) \times \rho} & N^{Q}
\end{array}\right], \quad Q^{-1} \widehat{G}=\left[\begin{array}{c}
\widehat{G}_{1}^{Q} \\
0_{\left(m n_{r}-\rho\right) \times r_{1}}
\end{array}\right],
$$

где $\widehat{D}_{1}^{Q} \in \mathbb{R}^{\rho \times \rho}, \widehat{G}_{1}^{Q} \in \mathbb{R}^{\rho \times r_{1}}$, размеры блоков $\widehat{D}_{2}^{Q}, N^{Q}$ очевидны. Непосредственной проверкой можно убедиться в том, что

$$
\operatorname{rank}\left[\widehat{G}_{1}^{Q}, \widehat{D}_{1}^{Q} \widehat{G}_{1}^{Q}, \ldots,\left(\widehat{D}_{1}^{Q}\right)^{\rho-1} \widehat{G}_{1}^{Q}\right]=\rho
$$

Заметим, что в силу равенства (14) блок $N^{Q}$ нильпотентный, следовательно,

$$
\operatorname{det}\left[E_{m n_{r}-\rho}-N^{Q} \lambda\right] \equiv 1
$$

Из равенства (16) следует существование матрицы $L_{1}^{Q} \in \mathbb{R}^{r_{1} \times \rho}$ такой, что

$$
\operatorname{det}\left[E_{\rho}-\left(\widehat{D}_{1}+\widehat{G}_{1}^{Q} L_{1}^{Q}\right) \lambda\right] \equiv 1
$$

Для ее построения достаточно привести матрицу $\widehat{D}_{1}^{Q}$ к блочно-треугольному виду с диагональными блоками в форме Фробениуса. Положим $L^{x y}=\left[L_{1}^{Q}, L_{2}^{Q}\right] Q^{-1}$, где $L_{2}^{Q} \in \mathbb{R}^{r_{1} \times\left(m n_{r}-\rho\right)}-$ любая матрица. Тогда

$$
\operatorname{det}\left[E_{m n_{r}}-\left(\widehat{D}+\widehat{G} L^{x y}\right) \lambda\right]=\operatorname{det}\left[E_{\rho}-\left(\widehat{D}_{1}^{Q}+\widehat{G}_{1}^{Q} L_{1}^{Q}\right) \lambda\right] \operatorname{det}\left[E_{m n_{r}-\rho}-N^{Q} \lambda\right] \equiv 1 .
$$

В тождестве (11) положим $L^{x}(\lambda)=\sum_{j=1}^{m} L_{j}^{x} \lambda^{j}, L^{y}(\lambda)=\sum_{j=1}^{m} L_{j}^{y} \lambda^{j}$. Покажем, что определенные таким образом матрицы $L^{x}(\lambda), L^{y}(\lambda)$ обеспечивают выполнение тождества (11). Для этого проведем элементарные преобразования над определителем (15) и применим теорему Лапласа так, чтобы имела место следующая цепочка равенств:

$$
1 \equiv \operatorname{det}\left[E_{m n_{r}}-\left(\widehat{D}+\widehat{G} L^{x y}\right) \lambda\right]=
$$


(к блоку определителя, состоящего из первых $n$ строк, прибавим блок определителя, составленного из строк с номерами от $m n+1$ до $m n+r_{1}$ и умноженного слева на матрицу $G_{0}$ )

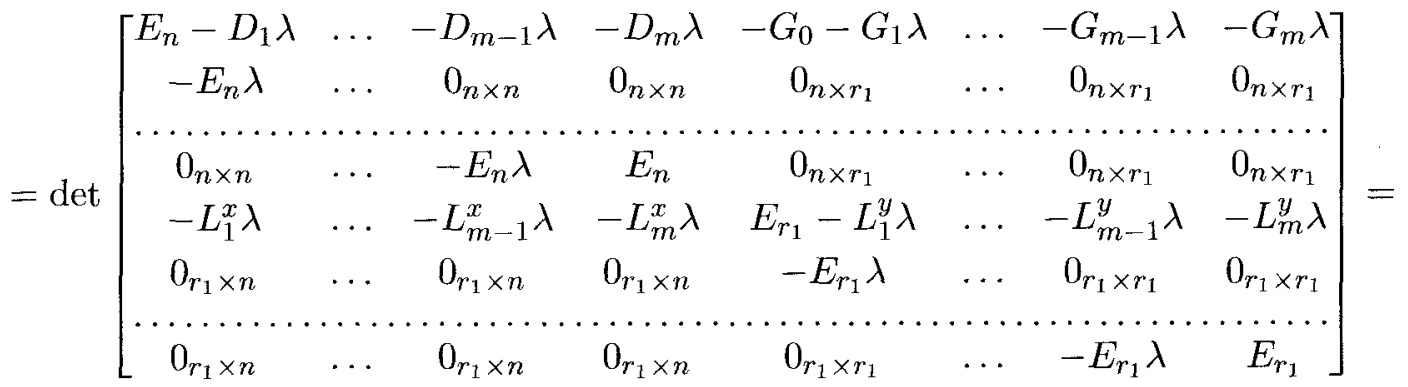

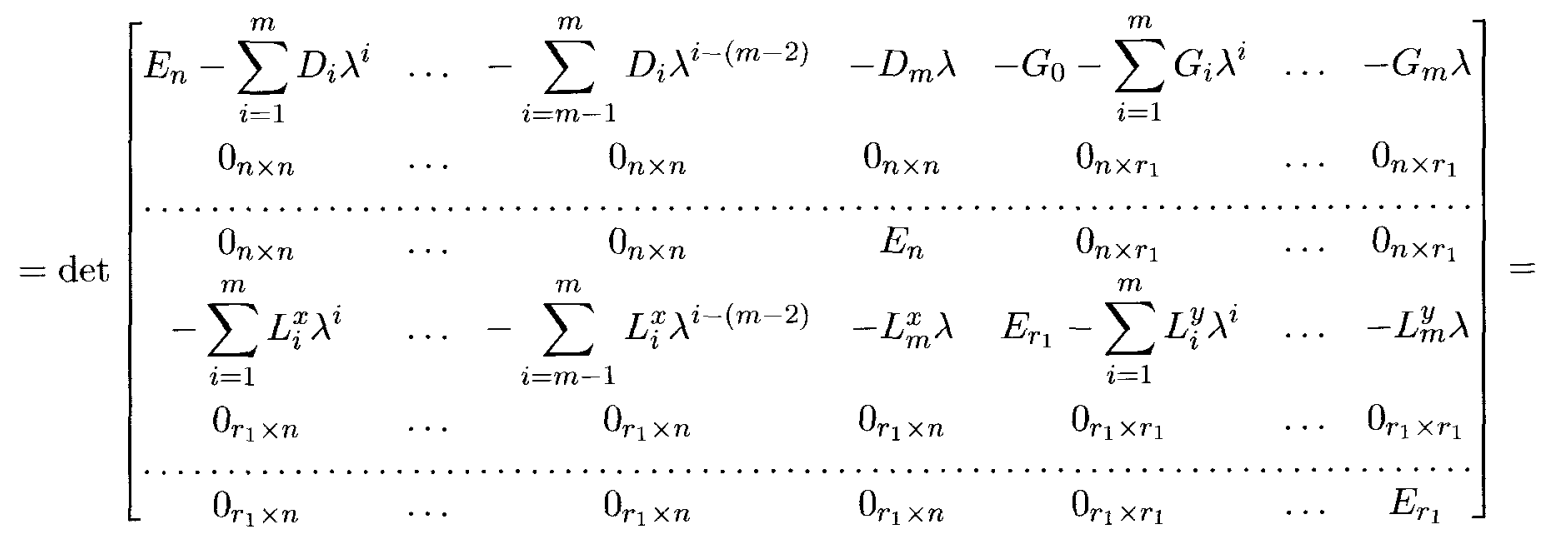

$$
\begin{aligned}
& =\operatorname{det}\left[\begin{array}{cc}
E_{n}-D(\lambda) & -G(\lambda) \\
-L^{x}(\lambda) & E_{r_{1}}-L^{y}(\lambda)
\end{array}\right] \text {. }
\end{aligned}
$$

Лемма доказана.

3.3. Синтез третьего контура. Третий контур регулятора строится так, чтобы у замкнутой системы (1), (3)-(5) вырождались первые компоненты ее фазового вектора, соответствующие компонентам исходной системы (1), т.е. выполнялось тождество (2). В данном случае удобнее выбрать матрицы $S^{u}(\lambda), S^{\psi}(\lambda), S_{2}(\lambda), S_{3}(\lambda)$ регулятора $(6)$, которые обеспечат системе $(10)$ свойство [17] точечной вырожденности в направлениях $g_{i}=\operatorname{col}[0, \ldots, 0,1,0, \ldots, 0]$, $i=\overline{1, n_{r}+s-1}$ (где единица стоит на $i$-м месте). Поэтому отдельно записывать матрицы, входящие в структуру третьего контура регулятора (3)-(5), нет необходимости.

Рассмотрим тройку матриц $\check{D}(\lambda)$,

$$
\check{A}(\lambda)=\left[\begin{array}{cc}
A(\lambda) & 0_{n \times r_{1}} \\
0_{r_{1} \times n} & 0_{r_{1} \times r_{1}}
\end{array}\right], \quad \bar{B}(\lambda)=\left[\begin{array}{cc}
G(\lambda) & 0_{n \times r_{1}} \\
0_{r_{1} \times r_{1}} & E_{r_{1}}
\end{array}\right] .
$$

Из условий (8), (11) следует, что

$$
\operatorname{rank}\left[p\left(E_{n_{r}}-\check{D}\left(e^{-p h}\right)\right)-\check{A}\left(e^{-p h}\right), \bar{B}\left(e^{-p h}\right)\right]=n_{r}, \quad p \in \mathbb{C} .
$$

Обозначим через $\Pi(\lambda)$ матрицу, обратную матрице $\left(E_{n_{r}}-\check{D}(\lambda)\right), \bar{A}(\lambda)=\check{A}(\lambda) \Pi(\lambda)$. В силу тождества (11) матрица П $(\lambda)$ унимодулярная, поэтому из равенства (17) следует, что

$$
\operatorname{rank}\left[p E_{n_{r}}-\bar{A}\left(e^{-p h}\right), \bar{B}\left(e^{-p h}\right)\right]=n_{r}, \quad p \in \mathbb{C} .
$$

Пусть $\bar{B}(\lambda)=\left[\bar{b}_{1}(\lambda), \ldots, \bar{b}_{2 r_{1}}(\lambda)\right]$. Выберем любые $\theta \quad\left(\theta \leq 2 r_{1}\right)$ столбцов матрицы $\bar{B}(\lambda)$ так, чтобы выполнялись следующие равенства:

$$
\operatorname{rank}\left[\bar{b}_{s_{1}}(\lambda), \ldots, \bar{A}^{n_{1}-1}(\lambda) \bar{b}_{s_{1}}(\lambda)\right]=n_{1},
$$




$$
\begin{gathered}
\operatorname{rank}\left[\bar{b}_{s_{1}}(\lambda), \ldots, \bar{A}^{n_{1}-1}(\lambda) \bar{b}_{s_{1}}(\lambda), \bar{A}^{n_{1}}(\lambda) \bar{b}_{s_{1}}(\lambda)\right]=n_{1}, \\
\operatorname{rank}\left[\bar{b}_{s_{1}}(\lambda), \ldots, \bar{A}^{n_{1}-1}(\lambda) \bar{b}_{s_{1}}(\lambda), \bar{b}_{s_{2}}(\lambda), \ldots, \bar{A}^{n_{2}-1}(\lambda) \bar{b}_{s_{2}}(\lambda)\right]=n_{1}+n_{2}, \\
\operatorname{rank}\left[\bar{b}_{s_{1}}(\lambda), \ldots, \bar{A}^{n_{1}-1}(\lambda) \bar{b}_{s_{1}}(\lambda), \bar{b}_{s_{2}}(\lambda), \ldots, \bar{A}^{n_{2}-1}(\lambda) \bar{b}_{s_{2}}(\lambda), \bar{A}^{n_{2}}(\lambda) \bar{b}_{s_{2}}(\lambda)\right]=n_{1}+n_{2}, \quad \ldots, \\
\operatorname{rank}\left[\bar{b}_{s_{1}}(\lambda), \ldots, \bar{A}^{n_{1}-1}(\lambda) \bar{b}_{s_{1}}(\lambda), \bar{b}_{s_{2}}(\lambda), \ldots, \bar{A}^{n_{2}-1}(\lambda) \bar{b}_{s_{2}}(\lambda), \ldots, \bar{b}_{s_{\theta}}(\lambda), \ldots, \bar{A}^{n_{\theta}-1}(\lambda) \bar{b}_{s_{\theta}}(\lambda)\right]= \\
=n_{1}+\ldots+n_{\theta}=n_{r} .
\end{gathered}
$$

Обозначим

$$
\bar{B}_{\bar{A}}(\lambda)=\left[\bar{b}_{s_{1}}(\lambda), \ldots, \bar{A}^{n_{1}-1}(\lambda) \bar{b}_{s_{1}}(\lambda), \bar{b}_{s_{2}}(\lambda), \ldots, \bar{A}^{n_{2}-1}(\lambda) \bar{b}_{s_{2}}(\lambda), \ldots, \bar{b}_{s_{\theta}}(\lambda), \ldots, \bar{A}^{n_{\theta}-1}(\lambda) \bar{b}_{s_{\theta}}(\lambda)\right] .
$$

Учитывая, что $\operatorname{rank} \bar{B}_{\bar{A}}(\lambda)=n_{r}$, построим квадратную полиномиальную матрицу $F(\lambda)$, $\operatorname{det} F(\lambda) \equiv$ const $\neq 0$, такую, что матрица $F(\lambda) \bar{B}_{\bar{A}}(\lambda)$ будет иметь следующую структуру:

$$
F(\lambda) \bar{B}_{\bar{A}}(\lambda)=\left[\begin{array}{cccc}
0 & 0 & \ldots & * \\
\ldots & \ldots & \ldots & \ldots \\
0 & * & \ldots & * \\
* & * & \ldots & *
\end{array}\right]
$$

где звездочкой обозначены некоторые полиномы, причем полиномы, стоящие на побочной диагонали, отличны от тождественного нуля. Заметим, что умножение матрицы $\bar{B}_{\bar{A}}(\lambda)$ слева на матрицу $F(\lambda)$ равносильно элементарным преобразованиям ее строк. Положим

$$
A_{F}(\lambda)=F(\lambda) \bar{A}(\lambda) F^{-1}(\lambda), \quad B_{F}(\lambda)=F(\lambda) \bar{B}(\lambda), \quad b_{F}(\lambda)=F(\lambda) \bar{b}_{s_{1}}(\lambda)
$$

и выберем вектор $\gamma \in \mathbb{R}^{2 r_{1}}$ такой, что $b_{F}(\lambda)=B_{F}(\lambda) \gamma$. В силу равенства (18) существует [3] матрица $K(\lambda) \in \mathbb{R}^{2 r_{1} \times n_{r}}[\lambda]$ такая, что

$$
\operatorname{rank}\left[p E_{n_{r}}-A_{K}\left(e^{-p h}\right), b_{F}\left(e^{-p h}\right)\right]=n_{r}, \quad p \in \mathbb{C},
$$

где $A_{K}(\lambda)=A_{F}(\lambda)+B_{F}(\lambda) K(\lambda)$.

Дальнейшие рассуждения будут более понятными, если ввести вспомогательную систему

$$
\dot{x}_{F}(t)=A_{K}(\lambda) x_{F}(t)+b_{F}(\lambda) z_{F}^{1}(t), \quad \dot{z}_{F}^{1}(t)=u_{F}(t), \quad t>0,
$$

где $\operatorname{col}\left[x_{F}, z_{F}^{1}\right]$ - решение системы $(20), u_{F}$ - скалярное управление. В силу равенства (19)

$$
\operatorname{rank}\left[\begin{array}{ccc}
p E_{n_{r}}-A_{K}\left(e^{-p h}\right) & -b_{F}\left(e^{-p h}\right) & 0 \\
0_{1 \times n_{r}} & p & 1
\end{array}\right]=n_{r}+1, \quad p \in \mathbb{C},
$$

т.е. система (20) является спектрально управляемой [1]. В работах [5-7] показано, что линейную автономную дифференциально-разностную систему с одним входом при наличии свойства спектральной управляемости всегда можно замкнуть линейной обратной связью, обеспечивающей ее точечную вырожденность в направлениях, отвечающих компонентам разомкнутой системы. Используя методику работы [5], замкнем систему (20) регулятором

$$
\begin{gathered}
u_{F}(t)=R_{11}(\lambda) x_{F}(t)+R_{12}(\lambda) z_{F}^{1}(t)+R_{13}(\lambda) z_{F}^{2}(t), \\
\dot{z}_{F}^{2}(t)=R_{21}(\lambda) x_{F}(t)+R_{22}(\lambda) z_{F}^{1}(t)+R_{23}(\lambda) z_{F}^{2}(t), \quad t>0,
\end{gathered}
$$

где

$$
\begin{gathered}
R_{11}(\lambda) \in \mathbb{R}^{1 \times n_{r}}[\lambda], \quad R_{12}(\lambda) \in \mathbb{R}^{1 \times 1}[\lambda], \quad R_{13}(\lambda) \in \mathbb{R}^{1 \times(s-1)}[\lambda] \\
R_{21}(\lambda) \in \mathbb{R}^{(s-1) \times n_{r}}[\lambda], \quad R_{22}(\lambda) \in \mathbb{R}^{(s-1) \times 1}[\lambda], \quad R_{23}(\lambda) \in \mathbb{R}^{(s-1) \times(s-1)}[\lambda]
\end{gathered}
$$


Параметры регулятора (21) выберем таким образом, чтобы замкнутая система (20), (21), т.е. система

$$
\left[\begin{array}{c}
\dot{x}_{F}(t) \\
\dot{z}_{F}^{1}(t) \\
\dot{z}_{F}^{2}(t)
\end{array}\right]=\left[\begin{array}{ccc}
A_{K}(\lambda) & \bar{b}_{F}(\lambda) & 0_{n_{r} \times(s-1)} \\
R_{11}(\lambda) & R_{12}(\lambda) & R_{13}(\lambda) \\
R_{21}(\lambda) & R_{22}(\lambda) & R_{23}(\lambda)
\end{array}\right]\left[\begin{array}{c}
x_{F}(t) \\
z_{F}^{1}(t) \\
z_{F}^{2}(t)
\end{array}\right], \quad t>0,
$$

стала точечно вырожденной [17] в направлениях $g_{i}, i=\overline{1, n_{r}+s-1}$, т.е. найдется момент времени $\bar{t}_{1}>0$ такой, что

$$
x_{F}(t) \equiv 0, \quad g_{i}^{\prime} \operatorname{col}\left[x_{F}(t), z_{F}^{1}(t), z_{F}^{2}(t)\right] \equiv 0, \quad t \geq \bar{t}_{1}, \quad i=\overline{n_{r}+1, n_{r}+s-1},
$$

каким бы ни было начальное условие системы (22). Кроме того, согласно [5] алгоритму построения регулятора (21), система (22) будет иметь конечный спектр, возможно, содержащий инвариантные спектральные значения [6]. Если такие значения отсутствуют, то спектр системы (22) и соответственно замкнутой системы (1), (6) может быть выбран произвольным. В случае комплексно-сопряженных инвариантных значений два последних уравнения регулятора (21) могут содержать [5] дробные запаздывания $h / \nu, \nu$ - некоторое натуральное число. Соответственно в операторную запись этих уравнений вместо $\lambda$ будет входить $\lambda^{k / \nu}$ : $\lambda^{k / \nu} f(t)=f(t-k h / \nu), \quad k$ - натуральное число.

Замечание 4. Если вектор $b_{F}(\lambda)=b_{F}$ постоянный, то паре матриц $\left\{A_{K}(\lambda), b_{F}\right\}$ можно поставить в соответствие систему вида (20) размера $n_{r}$ невырожденным преобразованием переменных с постоянной матрицей $U$ такой, что $U b_{F}=\operatorname{col}[0, \ldots, 0,1]$.

Рассмотрим систему (10) и регулятор (6). Положим

$$
\begin{gathered}
S_{\psi}^{u}(\lambda)=\left[E_{r_{1}}, 0_{r_{1} \times r_{1}}\right]\left[K(\lambda) F(\lambda)\left(E_{n_{r}}-\check{D}(\lambda)\right), \gamma, 0_{2 r_{1} \times(s-1)}\right], \\
S^{u}(\lambda)=\left[E_{r}, 0_{r \times r_{T}}\right] S_{\psi}^{u}(\lambda), \quad S^{\psi}(\lambda)=\left[0_{r_{T} \times r}, E_{r_{T}}\right] S_{\psi}^{u}(\lambda), \\
S_{2}(\lambda)=\left[0_{r_{1} \times r_{1}}, E_{r_{1}}\right]\left[K(\lambda) F(\lambda)\left(E_{n_{r}}-\check{D}(\lambda)\right), \gamma, 0_{2 r_{1} \times(s-1)}\right], \\
S_{3}(\lambda)=\left[\begin{array}{lll}
R_{11}(\lambda) F(\lambda)\left(E_{n_{r}}-\check{D}(\lambda)\right) & R_{12}(\lambda) & R_{13}(\lambda) \\
R_{21}(\lambda) F(\lambda)\left(E_{n_{r}}-\check{D}(\lambda)\right) & R_{22}(\lambda) & R_{23}(\lambda)
\end{array}\right] .
\end{gathered}
$$

3.4. Обоснование точечной вырожденности системы (10). Как показано выпе, решение $X(t), t>m h$, системы $(1),(6)$ определяется системой (10). Поэтому если у системы (10) будут вырождаться не менее $n$ первых компонент, то это значит, что регулятор (6) обеспечивает выполнение тождества (2).

Утверждение 1. Система (10) лвляется точечно вырожденной в направлениях $g_{i}$, $i=\overline{1, n_{r}+s-1}$.

Доказательство. Систему (22) запишем в виде

$$
\dot{\bar{X}}(t)=\bar{A}(\lambda) \bar{X}(t), \quad t>0,
$$

где $\bar{X}(t)=\operatorname{col}\left[x_{F}(t), z_{F}^{1}(t), z_{F}^{2}(t)\right]$, а матрица $\bar{A}(\lambda)$ очевидным образом определяется из системы $(22)$. Обозначим $\bar{F}(\lambda)=\operatorname{diag}\left[F(\lambda), E_{s}\right]$ и заменой переменных $\bar{X}(t)=\bar{F}(\lambda) \tilde{X}(t)$ систему (24) приведем к виду

$$
\dot{\tilde{X}}(t)=\tilde{A}(\lambda) \tilde{X}(t), \quad t>0,
$$

где $\tilde{A}(\lambda)=\bar{F}^{-1}(\lambda) \bar{A}(\lambda) \bar{F}(\lambda)$. Обратим внимание, что первые $n_{r}+s-1$ компонент вектора $\tilde{X}(t)$ при $t \geq \bar{t}_{2} \geq \bar{t}_{1}\left(\bar{t}_{2}>0\right.$ - некоторый момент времени) тождественно равны нулю. Положив $\tilde{X}(t)=\operatorname{diag}\left[E_{n_{r}}-\tilde{D}(\lambda), E_{s}\right] X(t)$, получим систему $(10)$, первые $n_{r}+s-1$ компонент которой удовлетворяют тождествам

$$
\left[E_{n_{r}}-\check{D}(\lambda)\right] \operatorname{col}[x(t), y(t)] \equiv 0, \quad g_{i}^{\prime} X(t) \equiv 0, \quad t>t_{2}, \quad i=\overline{n_{r}+1, n_{r}+s-1}
$$


где $t_{2}=\min \left\{\bar{t}_{2}, m h\right\}$. Представим матрицу $\check{D}(\lambda)$ в виде $\check{D}(\lambda)=\sum_{i=1}^{m} \check{D}_{i} \lambda^{i}, \check{D}_{i}$ - некоторые матрицы. Обозначим

$$
\begin{gathered}
\breve{x}(t)=\operatorname{col}[x(t), y(t), \ldots, x(t-(m-1) h), y(t-(m-1) h)], \\
\tilde{D}=\left[\begin{array}{cccc}
\check{D}_{1} & \ldots & \check{D}_{m-1} & \check{D}_{m} \\
E_{n_{r}} & \ldots & 0_{n_{r} \times n_{r}} & 0_{n_{r} \times n_{r}} \\
\ldots \ldots \ldots \ldots & \ldots \ldots \ldots \ldots \ldots \\
0_{n_{r} \times n_{r}} & \ldots & E_{n_{r}} & 0_{n_{r} \times n_{r}}
\end{array}\right]
\end{gathered}
$$

На основании первого из тождеств (26) заключаем, что функция $\breve{x}$ удовлетворяет разностному уравнению

$$
\breve{x}(t)=\tilde{D} \breve{x}(t-h), \quad t \geq t_{2} .
$$

Используя теорему Лапласа для определителей и тождество (11), доказываем равепство $\operatorname{det}\left[E_{m n_{r}}-\tilde{D} \lambda\right] \equiv 1$, т.е. матрица $\tilde{D}$ нильпотентная. Обозначим через $\xi$ индекс нильпотентности матрицы $\tilde{D}\left(\tilde{D}^{\xi}=0_{m n_{r} \times m n_{r}}\right)$. Из системы $(25)$ в силу нильпотентности матрицы $\tilde{D}$ следует, что $\breve{x}(t)=\tilde{D}^{\xi} \breve{x}(t-h)=0, t \geq t_{2}+(\xi-1) h$. Доказательство завершаем использованием второго тождества из (26). Утверждение доказано.

Замечание 5. Поскольку система (10) имеет конечный спектр (совпадающий со спектром системы (22)), то функции $x(t), y(t), z(t)$ с течением времени сглаживаются. Это является существенным при построении непрерывной операции восстановления текущего состояния $X$ системы (10) $[13,14]$.

\section{4. ОБСУЖДЕНИЕ РЕЗУЛЬТАТОВ}

В работе получены условия существования и метод построения регулятора с обратной связью по состоянию, обеспечивающего успокоение решения исходной (разомкнутой) системы, а также конечный спектр и нейтральный тип замкнутой системы. Из работы [13] следует, что ослабить условия суцествования регулятора, обеспечивающего выполнение тождества (2), за счет смены его типа невозможно. Остановимся на некоторых моментах построения регулятора и приведем пример.

1. В работе [16] приведены условия, при которых для пары матриц $\{D(\lambda), G(\lambda)\}$ существует полиномиальная матрица $L(\lambda) \in \mathbb{R}^{r_{1} \times n}[\lambda], L(0)=0_{r_{1} \times n}$, такая, что

$$
\operatorname{det}\left[E_{n}-(D(\lambda)+G(\lambda) L(\lambda))\right] \equiv 1
$$

Данные условия являются более жесткими по сравнению с условием (9), однако если они выполняются, то размер регулятора (6) можно уменьшить. Для этого второй контур надо записать в виде $v(t)=L(\lambda) \dot{x}(t)+w(t)$. При этом регулятор (6) примет вид

$$
\begin{gathered}
u(t)=L_{1}(\lambda) \dot{x}(t)+S^{u}(\lambda) X(t)+T \psi(t), \quad \psi(t)=S \psi(t-h)+L_{2}(\lambda) \dot{x}(t)+S^{\psi}(\lambda) X(t), \\
\dot{z}(t)=S_{3}(\lambda) X(t), \quad t>0,
\end{gathered}
$$

где $L(\lambda)=\operatorname{col}\left[L_{1}(\lambda), L_{2}(\lambda)\right], X=\operatorname{col}[x, z]$. Чтобы уменынить размер второго контура регулятора, целесообразно рассмотреть отдельно соответствующие блоки пары матриц $\{D(\lambda), G(\lambda)\}$ и к некоторым из них применить результаты работы [16], а к некоторым - рассуждения п. 3.2. Такую ситуацию иллюстрирует приведенный ниже пример 2.

2. При выполнении условий (8), (9) можно наряду с тождеством (2) и конечным спектром обеспечить замкнутой системе (10) еще и асимптотическую (которая в силу конечности спектра влечет за собой экспоненциальную) устойчивость. Для этого рассмотрим систему (20). Поскольку для данной системы выполняется условие полной (спектральной) управляемости, ее можно $[6,7]$ замкнуть интегральной обратной связью так, чтобы обеспечить не только 
выполнение тождества (23) и конечный спектр, но и асимптотическую устойчивость (см. работы $[6,7])$. Взяв эту обратную связь вместо регулятора (21), получим аналог регулятора (6), содержащий в своей структуре интегральные составляющие, но лишь от одной вспомогательной переменной.

3. В структуру регулятора (6) входит алгебраическая связь, определяющая динамику изменения функции $\psi$. Поэтому входное воздействие $u$ на каждом полуинтервале $(k h,(k+1) h]$, $k=0,1, \ldots$, меняет свою сгруктуру как функцию состояния замкнутой системы. Можно показать [8], что если для системы (1) не выполняются условия полной управляемости (см. замечание 2), то построить регулятор постоянной структуры невозможно. Однако если система (1) обладает свойством полной управляемости, то ситуация меняется на противоположную.

4. Пусть система (1) обладает свойс'гвом полной управляемости. Тогда построение регулятора можно начинать сразу со второго контура, т.е. положить $u=v$. В результате регулятор (6) примет вид

$u(t)=\dot{y}(t)+S^{u}(\lambda) X(t), \quad \dot{y}(t)=L^{x}(\lambda) \dot{x}(t)+L^{y}(\lambda) \dot{y}(t)+S_{2}(\lambda) X(t), \quad \dot{z}(t)=S_{3}(\lambda) X(t), \quad t>0$.

5. Пусть система (1) обладает свойством полной управляемости и $B_{0} \neq 0, B_{i}=0, i=\overline{1, m}$, т.е. управление не содержит запаздывание. Тогда $[16]$ существует матрица $L(\lambda) \in \mathbb{R}^{r \times n}[\lambda]$, $L(0)=0_{r \times n}$, такая, что $\operatorname{det}\left[E_{n}-\left(D(\lambda)+B_{0} L(\lambda)\right)\right] \equiv 1$. В этом случае во втором контуре можно сразу положить $v(t)=L(\lambda) \dot{x}(t)+w(t)$ и перейти к построению третьего контура. В результате получим регулятор вида

$$
u(t)=L(\lambda) \dot{x}(t)+S_{2}(\lambda) X(t), \quad \dot{z}(t)=S_{3}(\lambda) X(t), \quad t>0,
$$

где $X=\operatorname{col}[x, z]$.

Пример 2. Рассмотрим систему (1) с матрицами $(m=2, n=2, h=\ln 2)$

$$
D(\lambda)=\left[\begin{array}{cc}
\lambda & 0 \\
-\lambda^{2} & \lambda
\end{array}\right], \quad A(\lambda)=\left[\begin{array}{cc}
0 & 0 \\
1-\lambda^{2} & 0
\end{array}\right], \quad B(\lambda)=\left[\begin{array}{cc}
1-\lambda & 0 \\
0 & \lambda^{2}
\end{array}\right]
$$

В данном случае нарушаются два условия полной управляемости, указанные в замечании 2. Строим [10] матрицы $T_{1}=\left[\begin{array}{ll}0 & 1 \\ 0 & 0\end{array}\right], \quad T_{2}=T=\left[\begin{array}{ll}1 & 0 \\ 0 & 0\end{array}\right]$ (в общем случае они определяются неоднозначно), $S=\left[\begin{array}{ll}0 & 1 \\ 0 & 1\end{array}\right], \quad \tilde{B}_{0}=\left[\begin{array}{ll}1 & 0 \\ 0 & 0\end{array}\right], \quad \tilde{B}_{1}=\left[\begin{array}{cc}-1 & 1 \\ 0 & 0\end{array}\right], \quad \tilde{B}(\lambda)=\left[\begin{array}{cc}1-\lambda & \lambda \\ 0 & 0\end{array}\right]$. Проверка показывает, что условия теоремы 1 выполняются. Перейдем к построению регулятора, обеспечивающего выполнение тождества (2).

а) Синтез первого контура. Положим

$$
u(t)=\left[\begin{array}{ll}
1 & 0 \\
0 & 0
\end{array}\right] \psi(t)+v^{1}(t), \quad \psi(t)=\left[\begin{array}{ll}
0 & 1 \\
0 & 1
\end{array}\right] \psi(t-\ln 2)+v^{2}(t)
$$

Замкнув исходную систему первым контуром регулятора, согласно лемме 1 , получим систему с новым входом $[B(\lambda), \tilde{B}(\lambda)] \operatorname{col}\left[v^{1}(t), v^{2}(t)\right]$, где $\operatorname{col}\left[v^{1}(t), v^{2}(t)\right]$ - новое управление. Матрица $[B(\lambda), \tilde{B}(\lambda)]=\left[\begin{array}{cccc}1-\lambda & 0 & 1-\lambda & \lambda \\ 0 & \lambda^{2} & 0 & 0\end{array}\right]$ имеет "избыточное" число столбцов, поэтому последующие вычисления можно упростить, введя новое управление. Пусть

$$
v^{1}(t)=\operatorname{col}\left[v_{1}^{1}(t), v_{2}^{1}(t)\right], \quad v^{2}(t)=\operatorname{col}\left[v_{1}^{2}(t), v_{2}^{2}(t)\right]
$$

Положим $v_{1}^{1}(t) \equiv 0, \quad v_{2}^{1}(t)=v_{2}(t), v^{2}(t)=\left[\begin{array}{cc}1 & 0 \\ 1 & 1\end{array}\right]\left[\begin{array}{c}v_{1}(t) \\ 0\end{array}\right]$. Тогда новый (упрощенный) вход системы примет вид $\left[\begin{array}{cc}1 & 0 \\ 0 & \lambda^{2}\end{array}\right]\left[\begin{array}{l}v_{1}(t) \\ v_{2}(t)\end{array}\right]$. В соответствии с замечанием 3 возьмем $G(\lambda)=\left[\begin{array}{cc}1 & 0 \\ 0 & \lambda^{2}\end{array}\right]$. 
б) Синтез второго контура. Пара матриц $\{D(\lambda), G(\lambda)\}$ имеет блочно-треугольную форму. поэтому обеспечить системе $(1),(3),(4)$ запаздывающий тип можно, рассмотрев отдельно соответствующие блоки, что позволит уменьшить размер второго контура. Для левых верхних блоков $\{\lambda, 1\}$ указанной пары положим $v_{1}(t)=-\dot{x}_{1}(t-\ln 2)+w_{1}(t)$. Для второй пары блоков $\left\{\lambda, \lambda^{2}\right\}$ находим (согласно доказательству леммы 2) $L^{x}(\lambda)=-\lambda, L^{y}(\lambda)=-\lambda-\lambda^{2}$ такие, что $\operatorname{det}\left[\begin{array}{cc}1-\lambda & -\lambda^{2} \\ \lambda & 1+\lambda+\lambda^{2}\end{array}\right] \equiv 1$. Теперь второй контур можно записать в виде

$$
\begin{gathered}
{\left[\begin{array}{l}
v_{1}(t) \\
v_{2}(t)
\end{array}\right]=\left[\begin{array}{ccc}
-\lambda & 0 & 0 \\
0 & 0 & 1
\end{array}\right]\left[\begin{array}{l}
\dot{x}(t) \\
\dot{y}(t)
\end{array}\right]+\left[\begin{array}{l}
w_{1}(t) \\
w_{2}(t)
\end{array}\right],} \\
\dot{y}(t)=[0,-\lambda] \dot{x}(t)+\left(-\lambda-\lambda^{2}\right) \dot{y}(t)+w_{3}(t) .
\end{gathered}
$$

Замкнув исходную систему первым и вторым контурами регулятора и применив лемму 1 , непосредственной проверкой убеждаемся в том, что полученная система имеет характеристический квазиполином запаздывающего типа.

в) Синтез третьего контура. Из вида полученной системы запаздывающего типа следует, что

$$
E_{3}-\check{D}(\lambda)=\left[\begin{array}{ccc}
1 & 0 & 0 \\
\lambda^{2} & 1-\lambda & -\lambda^{2} \\
0 & \lambda & 1+\lambda+\lambda^{2}
\end{array}\right], \quad \check{A}(\lambda)=\left[\begin{array}{ccc}
0 & 0 & 0 \\
1-\lambda^{2} & 0 & 0 \\
0 & 0 & 0
\end{array}\right], \quad \bar{B}(\lambda)=\left[\begin{array}{ccc}
1 & 0 & 0 \\
0 & \lambda^{2} & 0 \\
0 & 0 & 1
\end{array}\right] .
$$

Вычислив матрицы $\Pi(\lambda)$ и $\bar{A}(\lambda)$, выберем $\bar{B}_{\bar{A}}(\lambda)=\left[\bar{b}_{3}(\lambda), \bar{b}_{1}(\lambda), \bar{A}(\lambda) \bar{b}_{1}(\lambda)\right]$. Далее находим матрицы

$$
F(\lambda)=\left[\begin{array}{lll}
0 & 1 & 0 \\
1 & 0 & 0 \\
0 & 0 & 1
\end{array}\right], \quad A_{F}(\lambda), B_{F}(\lambda), K(\lambda)=\left[\begin{array}{lll}
0 & 0 & 1 \\
0 & 1 & 0 \\
0 & 0 & 0
\end{array}\right]
$$

вектор $\gamma=\operatorname{col}[0,0,1]$ и полагаем $b_{F}(\lambda)=b_{F}=\operatorname{col}[0,0,1]$.

В соответствии с замечанием 4 вместо системы (20) возьмем вспомогательную систему $\dot{x}_{F}(t)=A_{K}(\lambda) x_{F}(t)+b_{F} u_{F}(t), t>0$. Замкнем эту систему регулятором, который обеспечит вырождение первых трех компонент вектора решения замкнутой системы. Кроме того, у данной системы отсутствуют инвариантные собственные значения, поэтому замкнутой системе можно также обеспечить наперед заданный спектр, например, состоящий из чисел $\{-1,-2,-3,-4\}$. Используя методику работы [5], найдем указанный регулятор

$$
u_{F}(t)=\left[R_{11}(\lambda), R_{12}(\lambda)\right] \operatorname{col}\left[x_{F}(t), z_{F}^{1}(t)\right], \quad \dot{z}_{F}^{1}(t)=\left[R_{21}(\lambda), R_{22}(\lambda)\right] \operatorname{col}\left[x_{F}(t), z_{F}^{1}(t)\right]
$$

где $R_{11}(\lambda), R_{12}(\lambda), R_{21}(\lambda), R_{22}(\lambda)$ - полиномы не выше четвертой степени.

Далее, проведя синтез трех построенных контуров, окончательно получим регулятор вида

$$
\begin{aligned}
& u(t)=\left[\begin{array}{lll}
0 & 0 & 0 \\
0 & 0 & 1
\end{array}\right]\left[\begin{array}{l}
\dot{x}(t) \\
\dot{y}(t)
\end{array}\right]+\left[\begin{array}{llll}
0 & 0 & 0 & 0 \\
1 & 0 & 0 & 0
\end{array}\right] X(t)+\left[\begin{array}{ll}
1 & 0 \\
0 & 0
\end{array}\right] \psi(t)
\end{aligned}
$$

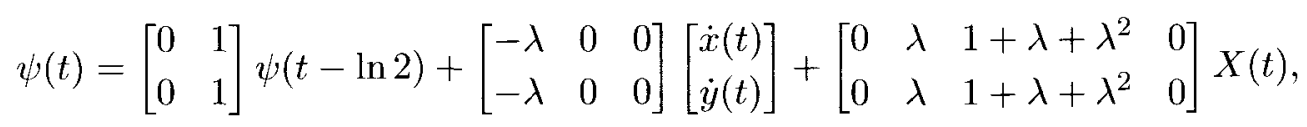

$$
\begin{aligned}
& \dot{y}(t)=[0,-\lambda] \dot{x}(t)+\left[-\lambda-\lambda^{2}\right] \dot{y}(t)+ \\
& +\left[\frac{-785}{21}+\frac{167 \lambda}{24}-\frac{31495 \lambda^{2}}{672}+\frac{6319 \lambda^{3}}{448}-\frac{77 \lambda^{4}}{48}+\frac{37 \lambda^{5}}{672}, \frac{-970}{21}+\frac{4183 \lambda}{84}-\frac{237 \lambda^{2}}{16}+\frac{533 \lambda^{3}}{336}-\frac{71 \lambda^{4}}{1344},\right. \\
& \left.\frac{24539 \lambda^{2}}{672}-\frac{5949 \lambda^{3}}{448}+\frac{687 \lambda^{4}}{448}-\frac{71 \lambda^{5}}{1344}-\frac{220}{21}-\frac{1613 \lambda}{168}, 1024-960 \lambda+280 \lambda^{2}-30 \lambda^{3}+\lambda^{4}\right] X(t),
\end{aligned}
$$




$$
\begin{gathered}
\dot{z}(t)=\left[\frac{-4765}{225792}+\frac{1481 \lambda}{903168}-\frac{81223 \lambda^{2}}{1806336}+\frac{1765 \lambda^{3}}{451584}-\frac{37 \lambda^{4}}{301056}, \frac{-5071}{112896}+\frac{20899 \lambda}{451584}-\frac{1741 \lambda^{2}}{451584}+\frac{71 \lambda^{3}}{602112}\right. \\
\left.\frac{25615 \lambda^{2}}{602112}-\frac{6751 \lambda^{3}}{1806336}+\frac{71 \lambda^{4}}{602112}-\frac{575}{225792}-\frac{2141 \lambda}{903168}, \frac{10}{21}-\frac{7 \lambda}{8}+\frac{7 \lambda^{2}}{96}-\frac{\lambda^{3}}{448}\right] X(t), \quad t>0
\end{gathered}
$$

Непосредственной проверкой [17] убеждаемся в том, что у системы (1), замкнутой построенным регулятором, вырождаются первые три компоненты, а ее спектр состоит из чисел $\{-1,-2,-3,-4\}$.

\section{СПИСОК ЛИТЕРАТУРЫ}

1. Manitius A., Triggiani $R$. Function space controllability of linear retarded systems: a derivation from abstract operator conditions // SIAM J. Control and Optimization. 1978. V. 16. № 4. P. 599-645.

2. Manitius A.Z., Olbrot A.W. Finite Spectrum Assignment Problem for Systems with Delays // IEEE Trans. on Autom. Control. 1979. AC-24. № 4. P. 541-553.

3. Watanabe $K$. Finite spectrum assignment and observer for multivariable systems with commensurate delays // IEEF Trans. on Autom. Control. 1986. V. 31. № 6. P. 543-550.

4. Watanabe K., Nobuyama E., Kitamori T. et al. A New Algorithm for Finite Spectrum Assignment of Single-Input Systems with Time Delay // IEEE Trans. on Autom. Control. 1992. AC-37. № 9. P. $1377-1383$.

5. Метельский A.B. Полное успокоение линейной автономной дифференциально-разностной системы регулятором того же типа // Дифференц. уравнения. 2012. Т. 48. № 9. С. 1240-1255.

6. Метельский A.B. Спектральное приведение, полное успокоение и стабилизация системы с запаздыванием одним регулятором // Дифференц. уравнения. 2013. Т. 49. № 11. С. 1436-1452.

7. Метельский A.B. Полное успокоение и стабилизация системы с запаздыванием через спектральное приведение // Изв. РАН. Теория и системы управления. 2014. № 1. С. 3-11.

8. Метельский A.В., Урбан О.И., Хартовский В.Е. Успокоение решения дифференциальных систем с многими запаздываниями посредством обратной связи / / Изв. РАН. Теория и системы управления. 2015. № 2. C. 40-50.

9. Красовский H.H. Оптимальные процессы в системах с запаздыванием // Статистические методы: Тр. II Междунар. конгресса ИФАК (Базель, 1963). М., 1965. Т. 2. С. 201-210.

10. Хартовский B.E. Обобщение задачи полной управляемости дифференциальных систем с соизмеримыми запаздываниями // Изв. РАН. Теория и системы управления. 2009. № 6. С. 3-11.

11. Хартовский B.E. Задача успокоения решения алгебро-дифференциальных вполне регулярных систем с последействием // Докл. НАН Беларуси. 2012. Т. 56. № 6. С. 5-11.

12. Хартовский B.E. Задача полной управляемости и ее обобщение для линейных автономных систем нейтрального типа // Изв. РАН. Теория и системы управления. 2012. № 6. С. 15-28.

13. Хартовский B.E., Павловская A.T. Полная управляемость и управляемость линейных автономных систем нейтрального типа // Автоматика и телемеханика. 2013. № 5. С. 59-79.

14. Метельский A.B., Минюк C.A. Критерии конструктивной идентифицируемости и полной управляемости линейных стационарных систем нейтрального типа с запаздыванием // Изв. РАН. Теория и системы управления. 2006. № 5. С. 15-23.

15. Хартовский B.E., Урбан О.И. Управление линейными автономными алгебро-дифференциальными системами посредством динамических регуляторов // Весці НАН Беларусі. Сер. фіз.-мат. навук. 2014. № 1. C. $36-42$.

16. Павловская A.T., Хартовский B.E. Управление линейными системами с запаздыванием нейтрального типа регуляторами с обратной связью динамической структуры // Изв РАН. Теория и системы управления. 2014. № 3. С. 3-18.

17. Метельский A.B. Проблема точечной полноты в теории управления дифференциально-разностными системами // Успехи мат. наук. 1994. Т. 49. Вып. 2(296). С. 103-141.

Белорусский национальный технический университет,

г. Минск,

Поступила в редакцию

Гродненский государственный университет

21.04 .2015 г.

им. Я. Купалы 